\title{
Clinical Presentation of Heart Failure Patients Admitted in National Institute of Cardiovascular Diseases, Dhaka
}

\author{
MD. TOUFIQUR RAHMAN, ${ }^{1}$ AAS MAJUMDER,${ }^{2}$ AFZALUR RAHMAN,${ }^{3}$ ABDUL WADUD CHOWDHURY ${ }^{4}$
}

\begin{abstract}
:
Background: Heart failure is a complex clinical syndrome that arises secondary to abnormalities of cardiac structure and/or function (inherited or acquired) that impair the ability of the left ventricle to fill or eject blood. The worldwide prevalence and incidence rates of heart failure (HF) are approaching epidemic proportions, as evidenced by the relentless increase in the number of HF hospitalizations, the growing number of HF-attributable deaths, and the spiraling costs associated with the care of HF patients. Worldwide, HF affects nearly 23 million people. In the United States, HF affects approximately 4.7 million persons ( 1.5 to 2 percent of the total population), with approximately 550,000 incident cases of HF diagnosed annually. Heart failure patients have various presentations and different etiologies. So, this study aimed to see the different clinical presentations of hospitalized heart failure patients.

Methods: This study was done to see Clinical Presentation of Heart Failure Patients admitted in National Institute of Cardiovascular Diseases, Dhaka. Total 2 I 12 patients were enrolled for this study during the period of August 2006 to July 2011.

Results: Most of the patients (65\%) were of 5 I-70 years age group. $75 \%$ (I584) patients were male. 98\% patients presented with SOB, $95 \%$ patients had basal crepitation, $74 \%$ had orthopnoea, $59 \%$ had Paroxysmal Nocturnal Dyspoea (PND), 40\% had leg edema and 25\% had raised JVP. Average heart rate was 85 beats/min, average systolic B.P. was $1 / 8 \mathrm{~mm} \mathrm{Hg}$ and average diastolic B.P. was $73 \mathrm{~mm} \mathrm{Hg}$. 45\% population had hypertension, 29\% patients had diabetes and $27 \%$ had concomitant respiratory illness. Average EF was $38 \%$. Ischemic Cardiomyopathy was the commonest (39\%) cause of heart failure, acute coronary syndrome was the second leading (29\%) cause and valvular heart disease is the third common cause.

Conclusion: Most of the heart failure patients are elderly age group. Most of the patients presented with shortness of breath and bilateral basal creps. Most patients had co-morbid other illness that influences the natural course of heart failure patients. Most common causes are ischemic cardiomyopathy, a sequel of ischemic insult of the heart. So, patients of acute or chronic ischemic heart diseases should be treated and follow up with care, considering their socioeconomic condition also.
\end{abstract}

Key words: Clinical presentation, Heart failure, hospitalized patients.

\section{Introduction:}

Bangladesh is passing through an epidemiological transition. Burden of infectious diseases are coming down while with increased life expectancy and wide spread change of lifestyle, non-communicable diseases are on the rise. ${ }^{1}$ Cardiovascular diseases are one of the main causes of morbidity and mortality in this country now. Heart failure (HF) is a significant

1. Associate Professor, Department of Cardiology, National Institute of Cardiovascular Diseases, Dhaka.

2. Director \& Professor, Department of Cardiology, National Institute of Cardiovascular Diseases, Dhaka.

3. Professor, Department of Cardiology, National Institute of Cardiovascular Diseases, Dhaka.

4. Associate Professor, Department of Cardiology, Dhaka Medical College \& Hospital.

Correspondence: Dr. Md. Toufiqur Rahman, Associate Professor, Dept. of Cardiology, Room No.225, North Block, National institute of Cardiovascular Diseases(NICVD), Dhaka. E-mail: drtoufiq19711@yahoo.com; drtoufiq1971@gmail.com and growing health problem as the population ages. Despite improvements in therapy, mortality and morbidity remain high. ${ }^{1,2}$

Heart failure is a complex clinical syndrome that arises secondary to abnormalities of cardiac structure and/or function (inherited or acquired) that impair the ability of the left ventricle to fill or eject blood. ${ }^{1}$

The worldwide prevalence and incidence rates of heart failure are approaching epidemic proportions, as evidenced by the relentless increase in the number of heart failure attributable death, and the spiraling costs associated with the care of heart failure patients. Worldwide, heart failure affects approximately 23 million people. In the United States, heart failure affects approximately 4.7 million persons (1.5 to 2 percent of the total population), with nearly 550000 incident cases of heart failure diagnosed annually. ${ }^{1,2}$

Estimates of the prevalence of symptomatic heart failure in the general European population are similar to those in the 
United States and range from 0.4 to 2 percent. The prevalence of heart failure follows an exponential pattern, rising with age, and affects 6 to 10 percent of people older than 65 years. The overall prevalence of heart failure is thought to be increasing, in part because our current therapies of cardiac disorders, such as myocardial infarction, valvular heart disease, and arrhythmias, are allowing patients to survive longer. ${ }^{2}$

Many of the typical signs and symptoms of heart failure are not directly attributable to the cardiac abnormalities that occur in the failing heart, but rather arise secondarily to abnormalities that occur in remote organs (e.g., kidney) or tissues (e.g., skeletal muscle). The dysfunction that occurs in these organs and tissues cannot be explained solely by decreased perfusion pressure, suggesting that other systemic processes (e.g., neurohormonal activation) must contribute to the syndrome of heart failure. Although heart failure was once thought to arise primarily in the setting of depressed left ventricular (LV) ejection fraction (EF), epidemiological studies have shown that approximately 50 percent of patients who develop heart failure have a normal or preserved EF. Epidemiological studies have also shown that patients may have significant abnormalities of LV contraction and relaxation and yet have no symptoms, in which case these patients are referred to as having asymptomatic heart failure. When a patient with chronic heart failure deteriorates, the patient is referred to as having decompensated or, alternatively, acute decompensated heart failure if the symptoms arise abruptly. Congestive heart failure is largely an outdated term that reflects the original clinical observation that patients with heart failure were frequently edematous or volume-overloaded. However, with current medical and device therapy, most patients with heart failure remain euvolemic, and are therefore referred to simply as having heart failure. ${ }^{2,3,4}$

In western developed countries, coronary artery disease, either alone or in combination with hypertension, seems to be the most common cause of heart failure. It is, however, very difficult to be certain what is the primary etiology of heart failure in a patient with multiple potential causes (for example, coronary artery disease, hypertension, diabetes mellitus, Atrial fibrillation, etc). Furthermore, even the absence of overt hypertension in a patient presenting with heart failure does not rule out an important etiological role in the past, with normalisation of blood pressure as the patient develops pump failure. The initial cohort of the Framingham heart study was monitored until 1965; hypertension appeared to be the most common cause of heart failure, being identified as the primary cause in $30 \%$ of men and $20 \%$ of women and a cofactor in a further $33 \%$ and $25 \%$, respectively. Moreover, electrocardiographic evidence of left ventricular hypertrophy in the presence of hypertension carried an approximate 15 fold increased risk of developing heart failure. In the subsequent years of follow up, however, coronary heart disease became increasingly prevalent before the development of heart failure and, as the identified cause of new cases of heart failure, increased from $22 \%$ in the $1950 \mathrm{~s}$ to almost $70 \%$ in the 1970 s.w1 11 During this period, the relative contribution of hypertension and valvular heart disease declined dramatically. ${ }^{5,6}$

There was an approximate 5\% and 30\% decline in the prevalence per decade of hypertension during this period among men and women, respectively. The declining contribution of hypertension most probably reflects the introduction of antihypertensive treatment; the parallel decline in the prevalence of left ventricular hypertrophy supports this supposition. It is also probable that during this same period, progressively greater accuracy in determining the presence of coronary heart disease contributed to its increasing importance in this regard. ${ }^{7-9}$

Framingham data has to consider the fact that heart failure was identified on clinical criteria alone and undoubtedly included individuals without associated left ventricular systolic dysfunction. Conversely, the large scale clinical trials have largely recruited patients who have reduced left ventricular ejection fractions and applied an extensive list of exclusion criteria. ${ }^{10-11}$

\section{Methods:}

The study population was the patients admitted in hospital with features of heart failure. Diagnostic criteria for diagnosis of congestive heart failure are paroxysmal nocturnal dyspnoea, orthopnea, exertional dyspnoea, elevated JVP, pulmonary basal crepitation, third heart sound, peripheral edema, night cough, hepatomegaly, pleural effusion, cardiomegaly on CXR, pulmonary edema on CXR, and echocardiographic findings.

Total 2112 patients were enrolled for the study during the period of August 2006 to July 2011.

Ethical approval for this study was granted by the ethics committee. A cardiologist took a standardized medical history and examined all the patients after hospital admission and the clinical findings of the admitting doctors were noted. Whenever possible an electrocardiogram, chest radiograph, transthoracic echocardiogram, serum biochemistry and haematology and thyroid function tests were performed. The echocardiogram was done to a standard protocol and 
according to accepted guidelines by a cardiologist and the two-dimensional, M-mode, Doppler and Colour-flow images were recorded.

\section{Results:}

Table-I

Baseline Characteristics of study population $N=2112$.

\begin{tabular}{lc}
\hline Characteristics & \\
\hline Age range & $14-87$ years \\
Average age & $46 \pm 07$ years \\
Most of patients (75\%) & $51-70$ years \\
Male patients & $1584(75 \%)$ \\
Female patients & $528(25 \%)$ \\
Ejection fraction & $38 \%$ \\
Average LV dimension in Diastole & $57 \mathrm{~mm}$ \\
Average LV dimension in Systole & $45 \mathrm{~mm}$ \\
Average pulse/min & $85 \mathrm{beats} / \mathrm{min}$ \\
Average Systolic BP & $116.46 \mathrm{~mm} \mathrm{Hg}$ \\
Average Diastolic BP & $75.77 \mathrm{~mm} \mathrm{Hg}$ \\
\hline
\end{tabular}

Table-II

Associated co-morbid conditions of study population $N=2112$

\begin{tabular}{lc}
\hline Characteristics & Percentage (\%) \\
\hline Hypertension & $45 \%$ \\
Diabetes & $29 \%$ \\
Dyslipidemia & $20 \%$ \\
Atrial fibrillation & $09 \%$ \\
Respiratory Diseases & $27 \%$ \\
\hline
\end{tabular}

Table-III

Presenting Features of study population $N=2112$

\begin{tabular}{lc}
\hline Characteristics & Percentage (\%) \\
\hline Shortness of Breath & $98 \%$ \\
Bilateral Basal Creps & $95 \%$ \\
Orthopnoea & $74 \%$ \\
Paroxysmal Nocturnal Dysnoea & $59 \%$ \\
Leg edema & $40 \%$ \\
Raised JVP & $25 \%$ \\
\hline
\end{tabular}

Table-IV

Causes of Heart Failure of study population $N=2112$.

\begin{tabular}{lc}
\hline Characteristics & No. (\%) \\
\hline Acute coronary syndrome & $620(29.37 \%)$ \\
Chronic ischemic heart disease & $43(02.02 \%)$ \\
Ischemic cardiomyopathy & $802(38.03 \%)$ \\
Dilated Cardiomyopathy & $86(04.12 \%)$ \\
Postpartum Cardiomyopathy & $25(1.18 \%)$ \\
Hypertension & $177(08.39 \%)$ \\
Valvular heart disease & $322(15.29 \%)$ \\
Cor-pulmonalae & $06(0.28 \%)$ \\
Congenital heart disease & $25(1.24 \%)$ \\
Hypothyroidism & $03(0.14 \%)$ \\
Hyperthyroidism & $03(0.14 \%)$ \\
\hline
\end{tabular}

\section{Discussion:}

Total 2112 patients of heart failure were enrolled. Patients are of 14 to 87 years age range. Average age was $46 \pm 07$ years. Most of the patients (75\%) in 51-70 years age groups. In SOLVD clinical trial, ${ }^{12}$ mean age was 61 years. In DIG study (1997), ${ }^{13}$ RALES study, ${ }^{14}$ MERIT-HF study, ${ }^{15}$ ATLAS Study ${ }^{16}$ mean age was 64 years. M Kabiruzzaman et al ${ }^{22}$ showed mean age was 54 years.

The Hillingdon heart failure study evaluated the incidence and aetiology of heart failure in one district of west London, England using clinical and echocardiographic data and a case definition based on three cardiologists applying the ESC definition of heart failure. The median age at the time of diagnosis of heart failure was 76 years. The incidence of heart failure was significantly higher in men than women at all ages with an age-standardised ratio of 1.75 . The primary aetiologies were coronary heart disease $(36 \%)$, unknown (34\%), hypertension (14\%), valve disease $(7 \%)$, atrial fibrillation alone $(5 \%)$, and other $(5 \%){ }^{8}$

Mcmurray et $\mathrm{al}^{9}$ studied trends in hospitalization for heart failure in Scotland 1980-1990. They found seventy-eight percent of discharges were in persons aged $>65 y$ ears and $48 \%$ of discharges were male.

In our study, Male was $75 \%$ and Female was $25 \%$. In SOLVED clinical trial, male was $80 \%$ and female was $20 \%$. In DIG study $^{13}$ and MERIT-HF ${ }^{15}$ male was $78 \%$.

In this study $98 \%$ patients presented with shortness of breath, 74\% patients presented with orthopnea, 59\% presented with paroxysmal nocturnal dyspnoea (PND), $40 \%$ presented with ankle edema, $25 \%$ presented with raised JVP and 95 had bilateral basal crepitations. 
In the present study, as a co- morbid condition, $45 \%$ had history of Hypertension, 29\% had Diabetes, 27\% had Respiratory disease, $09 \%$ had Atrial Fibrillation. In SOLVD (1991) clinical trial, ${ }^{12} 42 \%$ had Hypertension, 26\% had Diabetes, $10 \%$ had Atrial Fibrillation. In MERIT-HF clinical Trial, ${ }^{15} 44 \%$ had Hypertension, $25 \%$ had Diabetes and $17 \%$ had Atrial Fibrillation.

In our study, 69.42\% diagnosed as Ischemic Heart Disease (Acute Coronary Syndrome 29.37\%; Ischemic Cardiomyopathy $38.03 \%$ and Chronic Ischemic Heart Disease $2.02 \%$. In SOLVD ${ }^{12}$ clinical trial $71 \%$ had ischemic cause of heart failure, in $\mathrm{DIG}^{13}$ study $70 \%$ had ischemic cause of heart failure, in MERIT- $\mathrm{HF}^{15} 66 \%$ had ischemic cause of heart failure. In ATLAS ${ }^{16}$ study, $64 \%$ had ischemic cause of heart failure. In RALES study, ${ }^{14} 54 \%$ had ischemic cause of heart failure.

$08.39 \%$ patients had hypertensive heart failure, In $\mathrm{DIG}^{13}$ (1997) study, 09\% had hypertensive heart failure and in ATLAS $^{16}$ study , 20\% had hypertensive heart failure.

In the present study, $15.29 \%$ had valvular cause of heart failure. In ATLAS study, 06\% had valvular cause of heart failure. In SPICE registry, ${ }^{17} 05 \%$ had valvular cause of heart failure.

In our study, 04.12\% was diagnosed as DCM (Dilated Cardiomyopathy) as a cause of heart failure. In SOLVD (1991) clinical trial $18 \%$ had DCM, in DIG study $15 \%$ had DCM as a cause of heart failure, in SOLVD ${ }^{18}$ registry $13 \%$ had DCM. In ATLAS study, 28\% had DCM as a cause of heart failure. In SPICE ${ }^{17}$ registry, $17 \%$ had DCM.

In Pakistan, Jafary et al studied 196 patients with mean age $61.2 \pm 12.8$ years with a high preponderance of males. All of them were suffering from systolic heart failure with LVEF d" $40 \%$, requiring hospital admission with more than $60 \%$ suffering from hypertension $(67.3 \%)$ and diabetes mellitus $(60.7 \%)$ and more than three-fourths havinga history of coronary artery disease in the past. ${ }^{23}$

In the United Kingdom, most patients admitted to hospital with heart failure are more than 65 years old. The prevalence of heart failure rises from around 1\% in the age group 50-59 years to between 5 and $10 \%$ of those aged $80-89$ years. Heart failure is frequently due to coronary artery disease. ${ }^{24}$

Seow et $\mathrm{al}^{25}$ studied 225 patients in Singapore with LVEF $<40 \%$, their mean age was $68.5 \pm 2.3$ years and more than $51.1 \%$ of the subjects were aged 70 years and more. The most common cause of HF was coronary heart disease $(85.5 \%)$. Co morbid medical conditions were prevalent in this cohort, with $83.5 \%$ having at least one co-morbid condition. Hypertension was the most prevalent co-morbid condition; affecting $60 \%$ of the patients; followed by diabetes mellitus (56.9\%).

Remes et $\mathrm{al}^{27}$ studied incidence of heart failure in 45- 74 year old inhabitant in four rural communities in Eastern Finland. The incidence rates of heart failure increased with age in both sexes. Coronary heart disease or hypertension was evident in $80 \%$ cases.

\section{Conclusion:}

Despite a decline in age adjusted mortality from coronary heart disease (CHD) in developed countries overall, the number of patients with chronic CHD is increasing. This is principally the result of two separate trends. Firstly, the proportion of elderly in the population is increasing rapidly and these subjects have the highest incidence of CHD and hypertension. Secondly, survival in those with coronary artery disease is improving. In particular, it has been shown that survival after acute myocardial infarction has increased notably over the past decade, at least in part because of better medical treatment. As coronary artery disease is the most powerful risk factor for heart failure it is likely that the aforementioned trends will lead to an increase its future prevalence. Chronic heart failure may, therefore, become a more common manifestation of chronic heart disease and contribute too many more deaths. ${ }^{19-21,26}$

In our study, most of the heart failure patients are elderly age group. Most common cause is ischemic cardiomyopathy, a sequel of ischemic insult of the heart. So, patients of acute and chronic ischemic heart disease patients should be treated and follow up with care, considering their socioeconomic condition also. NSAIDs, Steroids, and other fluid retaining drugs should be used cautiously in cardiac patients prone to develop heart failure.

The number of patients with heart failure is bound to rise at the premature age if appropriate measurement is not taken to manage risk factors and to increase public awareness. Clinical and Epidemiological study is needed to explore further details.

\section{Conflict of interest: None.}

\section{References:}

1. Ministry of Health and Family Welfare, Government of People's Republic of Bangladesh. Strategic plan for Surveillance and Prevention of Noncomunicable Disease in Bangladesh 2007-2010. 2007, Dhaka

2. McMurray JJ, Petrie MC, Murdoch DR, et al. Clinical epidemiology of heart failure: public and private health burden. Eur Heart J1998; 19: P9-16. 
3. Ho KK, Pinsky JL, Kannel WB, et al.The epidemiology of heart failure: The Framingham study. J Am Coll Cardiol 1993; 22: 6A-13A.

4. McDonagh TA, Morrison CE, Lawrence A, et al. Symptomatic and asymptomatic left-ventricular systolic dysfunction in an urban population. Lancet 1997; 350: 829-33.

5. Senni M, Tribouilloy CM, Rodeheffer RJ. Congestive heart failure in the community: a study of all incident cases in Olmsted County, Minnesota, in 1991. Circulation 1998; 98 : 2282-9.

6. Harlan WR, Oberman A, Grimm R, et al: Chronic congestive heart failure in coronary artery disease. Clinical criteria. Ann Intern Med 1977; 86: 133.

7. Vasan RS, Larson MG, Benjamin EJ, et al. Congestive heart failure in subjects with normal versus reduced left ventricular ejection fraction: prevalence and mortality in a populationbased cohort. J Am Coll Cardiol 1999; 33: 1948-55.

8. Cowie MR, Wood DA, Coats AJ, et al. Incidence and aetiology of heart failure; a population-based study. Eur Heart J 1999; 20: 421-8.

9. McMurray J, McDonagh T, Morrison CE, et al. Trends in hospitalization for heart failure in Scotland 1980-1990. Eur Heart J 1993; 14: 1158-62.

10. Haldeman GA, Croft JB, Giles WH, et al. Hospitalization of patients with heart failure: national hospital discharge survey 1985-1995. Am Heart J 1999; 137: 352-60.

11. Kannel WB, Ho KK, Thom T. Changing epidemiological features of cardiac failure. Eur Heart J 1994; 72: S3-9.

12. The SOLVD Investigators. Effect of enalapril on survival in patients with reduced left ventricular ejection fractions and congestive heart failure. N Engl J Med 1991; 325: 293-302.

13. The Digitalis Investigation Group. The effect of digoxin on mortality and morbidity in patients with heart failure. $\mathrm{N}$ Engl J Med 1997; 336: 525-33.

14. Pitt B, Zannad F, Remme WJ, et al. The effect of spironolactone on morbidity and mortality in patients with severe heart failure. Randomized aldactone evaluation study investigators. N Engl J Med 1999; 341: 709-17

15. MERIT Investigators. Effect of metoprolol CR/XL in chronic heart failure: metoprolol CR/XL randomised intervention trial in congestive heart failure (Merit-HF). Lancet 1999; 353: 2001-7.
16. Packer M, Poole-Wilson PA, Armstrong PW, et al. Comparative effects of low and high doses of the angiotensin converting enzyme inhibitor, lisinopril, on morbidity and mortality in chronic heart failure. Circulation 1999; 100: 2312-18.

17. Bart BA, Ertl G, Held P, et al. Contemporary management of patients with left ventricular systolic dysfunction. Results from the study of patients intolerant of converting enzyme inhibitors (SPICE) registry. Eur Heart J 1999; 20: 1182-90.

18. SOLVD Investigators. Natural history and patterns of current practice in heart failure. J Am Coll Cardiol 1993; 4A: 14A-19A.

19. AAS Majumder, MT Rahman, MS Islam, et al. Aetiological Diagnosis of Heart Failure in Hospitalized Patients. Cardiovasc. j. 2009; 2(1) : 32-36

20. Rosamund WD, Chambless LE, Folsom AR, et al. Trends in the incidence of myocardial infarction and in mortality due to coronary artery disease. N Engl J Med 1998; 339: 861-7.

21. Bonneux L, Barendregt JJ, Meeter K, et al. Estimating clinical morbidity due to ischaemic heart disease and congestive heart failure: the future rise of heart failure.Am J Public Health 1994; 84:20-8.

22. Kabiruzzaman M, Malik FN, Ahmed N, et al. Burden of Heart Failure Patients in a Tertiary Level Cardiac Hospital. J Bangladesh Coll Phys Surg 2010; 28: 24-29

23. Jafary FH, KumarM, Chandna IE. Prognosis of hospitalized new- onset systolic heart failure in Indo-Asians- A lethal problem. J Cardiac Fail 2007; 13:855-860.

24. Bloomfield P, Bradbury A, Grubb NR, Newby DE. 2006.Cardiovascular disease. In: Boon NA, Colledge NR, Walker BR, Hunter JAA (eds). Davidson's Principles and Practice of Medicine, 20th ed. Churchill Livingstone, Edinburgh, pp. 519-644.

25. Seow SC, Lee YP, Chan YH, et al. Heart Failure Mortality in South Asian Patients With Left Ventricular Systolic Dysfunction. J Cardiac Fail 2007; 13:476-481.

26. The Task Force for the Diagnosis and Treatment of Acute and Chronic Heart Failure 2008 of the European Society of Cardiology. ESC Guidelines for the diagnosis and treatment of acute and chronic heart failure 2008. European Journal of Heart Failure dol:10.1016/j.ejheat.2008.08.005

27. Remes J, Reunanen A, Aromaa A, Pyorala K. Incidence of heart failure in Eastern Finland: a population-based surveillance study. Eur Heart J 1992; 13: 588-93. 